\title{
Temperature Distributions in Piezoelectric Photothermal Spectroscopy
}

\author{
J. Zakrzewski • M. Maliński · K. Strzałkowski
}

Received: 21 April 2012 / Accepted: 7 August 2012 / Published online: 29 August 2012

(C) The Author(s) 2012. This article is published with open access at Springerlink.com

\begin{abstract}
Piezoelectric photothermal spectroscopy is a method in which the stress and strain of a sample due to the absorption of electromagnetic radiation is detected by a piezoelectric transducer. The temperature distribution in the sample is the basis to obtain the theoretical amplitude and phase of photothermal piezoelectric spectra. In contrast to microphone detection, which needs only the temperature at one of the sample surfaces, in the piezoelectric one, it is necessary to know the spatial temperature distribution. The distributions given by Blonskij and by the modified interferential model of Malinski are applied. The influence of defect states in a volume and at the surfaces on the character of the amplitude and phase piezoelectric spectra is analyzed. The comparison of these approximate models and the two-layer one of Fernelius is presented.
\end{abstract}

Keywords Photothermal spectroscopy $\cdot$ Piezoelectric detection $\cdot$ Semiconductors

\section{Introduction}

The photothermal piezoelectric (PP) technique is very sensitive and is considered to be a direct method for measurements of the optical, thermal, and recombination parameters of semiconductors [1-5]. In the case of PP spectroscopy, it gives complementary results to absorption and photoluminescence techniques.

J. Zakrzewski $(\varangle) \cdot$ K. Strzałkowski

Instytut Fizyki, Uniwersytet Mikołaja Kopernika, ul. Grudziądzka 5/7, 87-100 Toruń, Poland e-mail: jzakrzew@ fizyka.umk.pl

\section{Maliński}

Department of Electronics, Technical University of Koszalin, ul. Sniadeckich 2,

75-328 Koszalin, Poland 
The method takes advantage of processes that occur in solid-state samples heated by a periodically modulated beam of light. As a result of periodical heating, a spatial and temporal temperature field arises in the sample.

PP spectroscopy, although relatively easy and convenient experimentally, using a phase-sensitive method, is difficult for quantitative interpretation. The dependence of the amplitude and phase spectra of the signals on the value of the optical absorption coefficient is strongly nonlinear. An additional difficulty arises from the fact that analyzed crystals are often not perfect, what results in the necessity of the development of special numerical models for interpretation of the spectra. For proper interpretation of amplitude and phase spectra, one needs to know or simulate the temperature spatial distribution in the sample and the absorption dependence on energy incident on the sample.

The theory of PP response was advanced by Jackson and Amer [6], but they used a complicated mathematical apparatus and this restricted the use of the theory. Blonskij et al. [7] introduced a simpler approach and had proposed to apply it to determination of the thermal diffusivity of solids.

In piezoelectric detection, one can also apply the models which are the basis of microphone detection (photoacoustic spectroscopy), but in this case it is necessary to know the full spatial thermal distribution along the sample width contrary to the temperature of the surface as it is in microphone detection. The temperature distribution based on the idea of Bennett and Patty [8] was used before by Malinski [9]. One can also use the distribution proposed in the theory of Rosencwaig and Gersho [10] or the two-layer extension of Fernelius [11].

The next step after having the temperature distribution is to apply it to the expression for voltage of the piezoelectric transducer. In the 1D case ( $x$-axis is along the width of the sample), the temperature at the $y z$ plane is averaged.

The aim of the article is to show the application and comparison of the different thermal distributions in piezoelectric spectroscopy.

\section{Theoretical Models of Piezoelectric Detection}

\subsection{Blonskij’s Model}

The authors considered a cylindrical sample of thickness $l$, radius $R$, thermal conductivity $k$, density $\rho$, and volumetric specific heat $C$. The periodical modulated light beam impinges on the sample surface $x=l / 2$; the piezoelectric detector is situated on the nonilluminated side of the sample at $x=-l / 2$.

The temperature distribution in the sample can be found from the heat conduction equation:

$$
\Delta T-\sigma^{2} T=-\frac{I \beta}{k} \mathrm{e}^{-r^{2} / b^{2}} \mathrm{e}^{-\beta(l / 2-x)}
$$

where $\beta$ is the optical absorption coefficient, $b$ is the beam radius, $r^{2}=y^{2}+z^{2}$, $\sigma=\frac{\mathrm{i} \omega}{\alpha}$, and $\alpha=\frac{k}{\rho C}$ is the thermal diffusivity. The author took into consideration 
the average temperature at the $y z$ plane and got the following expression for the temperature distribution:

$$
\langle T\rangle=\frac{I \pi b^{2} \beta}{k \sigma\left(\beta^{2}-\sigma^{2}\right)} \frac{\beta \cosh (\sigma(l / 2+x))-\beta \mathrm{e}^{-\beta l} \cosh (\sigma(l / 2-x))-\sigma \mathrm{e}^{-\beta\left(\frac{l}{2}-x\right)} \sinh (\sigma l)}{\sinh (\sigma l)}
$$

Solving the thermoelastic problem using the thin-film plate theory $[12,13]$, they get the expression for the electric voltage at the piezoelectric transducer:

$$
\begin{aligned}
V & =\frac{e_{\mathrm{p}^{\mathrm{L}}}}{\epsilon S} 2 \alpha_{\mathrm{t}}\left(\left\langle T_{0}\right\rangle-\frac{l}{2}\langle\tau\rangle\right) \\
\left\langle T_{0}\right\rangle & =\frac{1}{l} \int_{-l / 2}^{l / 2} T(x) \mathrm{d} x \\
\langle\tau\rangle & =\frac{12}{l^{3}} \int_{-l / 2}^{l / 2} T(x) x \mathrm{~d} x
\end{aligned}
$$

where $L$ is the thickness of the transducer, $S$ is its surface area, $\alpha_{t}$ is the linear thermal expansion coefficient, and $e_{\mathrm{p}}$ is the piezomodulus.

\subsection{Interference Model}

The other model to obtain the thermal distribution in the sample was proposed by Malinski [9]. It is based on the idea of Bennett and Patty [8]. For the sample of thickness $l$, a periodical modulated light beam falls on the surface at $x=0$ and the piezoelectric detector is situated on the nonilluminated side of the sample at $x=l$. One does not solve the nonlinear heat equations, instead, the thermal waves initiated by the light absorbed between $x$ and $x+\mathrm{d} x$ are considered ( $x$ is the axis of the width of the sample).

The amplitude of the thermal wave has the form,

$$
A=\frac{\beta I_{0} \exp (-\beta x)}{4 k \sigma} \mathrm{d} x
$$

These thermal waves travel through the sample, and multiply reflecting between the sample-backing and sample-gas boundaries. One must take into account that the waves originally travel toward the gas $\left(A \mathrm{e}^{\sigma x}\right)$ and the waves travel toward the backing $\left(A \mathrm{e}^{-\sigma x}\right)$. Summing these geometric series, one gets an expression for the temperature distribution [9]:

$$
T(x)=\frac{\beta I_{0}}{2 k \sigma\left[1-R_{\mathrm{b}} R_{\mathrm{g}} \exp (-2 \sigma l)\right]}[M(x)+N(x)]
$$




$$
\begin{aligned}
M(x)= & \frac{[\exp (\sigma x)+\exp (-\sigma x)][\exp ((-\sigma-\beta) x)-\exp ((-\sigma-\beta) l)]}{\beta+\sigma} \\
& +\frac{\left.R_{\mathrm{b}} \exp (-2 \sigma l)[\exp (\sigma x)+\exp (-\sigma x)][\exp ((\sigma-\beta) x))-\exp ((\sigma-\beta) l)\right]}{\beta-\sigma} \\
N(x)= & \frac{\left[\exp (-\sigma x)+R_{\mathrm{b}} \exp (-2 \sigma l+\sigma x)\right][1-\exp ((-\sigma-\beta) x)]}{\beta+\sigma} \\
& +\frac{\left[\exp (-\sigma x)+R_{\mathrm{b}} \exp (-2 \sigma l+\sigma x)\right][1-\exp ((\sigma-\beta) x)]}{\beta-\sigma}
\end{aligned}
$$

Having the thermal distribution one must solve, as previously, the thermoelastic problem to get the expression for the electric voltage at the piezoelectric transducer [9]. Following the conditions of the Malinski model, the expression has the form,

$$
V=-\left(\frac{1}{l} \int_{0}^{l} T(x) \mathrm{d} x-\frac{1}{2 r^{2}} \int_{0}^{l}\left(\frac{l}{2}-x\right) T(x) \mathrm{d} x\right)
$$

where $l$ is the thickness of the sample and $r=l /(2 \sqrt{3})$

\section{Absorption in Semiconductors}

To predict the character of piezoelectric spectra, one must know the dependence of the absorption coefficient. Typical ones can be taken into account [14]:

$$
\begin{array}{ll}
\beta=\beta_{0} \exp \left(\frac{\left(E-E_{\mathrm{g}}\right) \gamma}{0.025}\right) & \text { for } E \leq E_{\mathrm{g}} \\
\beta=A_{0}\left(E-E_{\mathrm{g}}\right)^{1 / 2} & \text { for } E>E_{\mathrm{g}}
\end{array}
$$

The first equation describes Urbach tail thermal broadening (this is represented by parameter $\gamma$ ) of the absorption band observed for all direct electron-type transition semiconductors, the second one-the absorption band connected with the direct, bandto-band, electron transitions in semiconductors.

\section{Amplitude and Phase of Piezoelectric Spectra}

\subsection{Ideal Crystal}

Figure 1 presents the theoretical predictions for the (a) amplitude and (b) phase piezoelectric spectra in the rear mode [9] configuration for a sample of thickness $1 \mathrm{~mm}$, thermal diffusivity $0.05 \mathrm{~cm}^{2} \cdot \mathrm{s}^{-1}, E_{\mathrm{g}}=2.74 \mathrm{eV}$, and $\gamma=0.6$. Both the interference and Blonskij's models give the same results. At the rear mode, the samples are irradiated from one side and the signal is collected from the other. A characteristic peak in the sub-bandgap region in the amplitude spectrum is clearly visible. According to 
Fig. 1 Simulation of (a) amplitude and (b) phase piezoelectric spectra for a sample of thickness $1 \mathrm{~mm}$, thermal diffusivity of $0.05 \mathrm{~cm}^{2} \cdot \mathrm{s}^{-1}, E_{\mathrm{g}}=2.74 \mathrm{eV}$, and $\gamma=0.6$

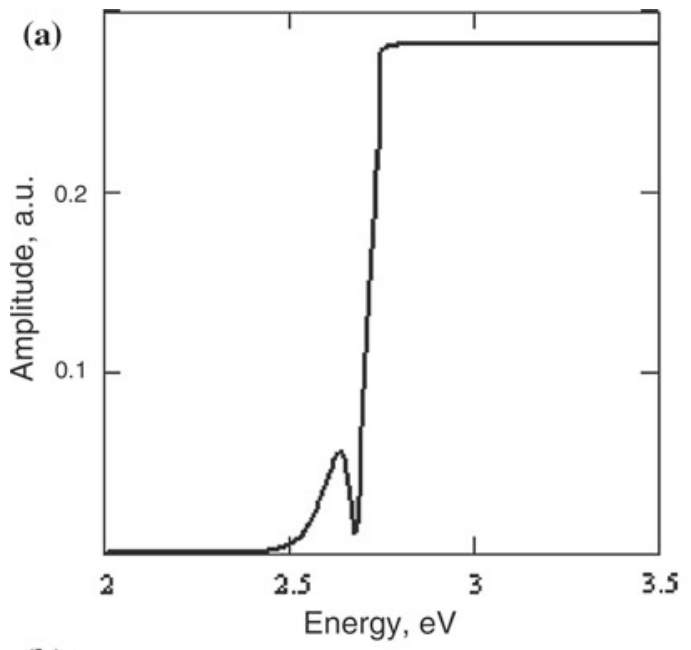

(b)

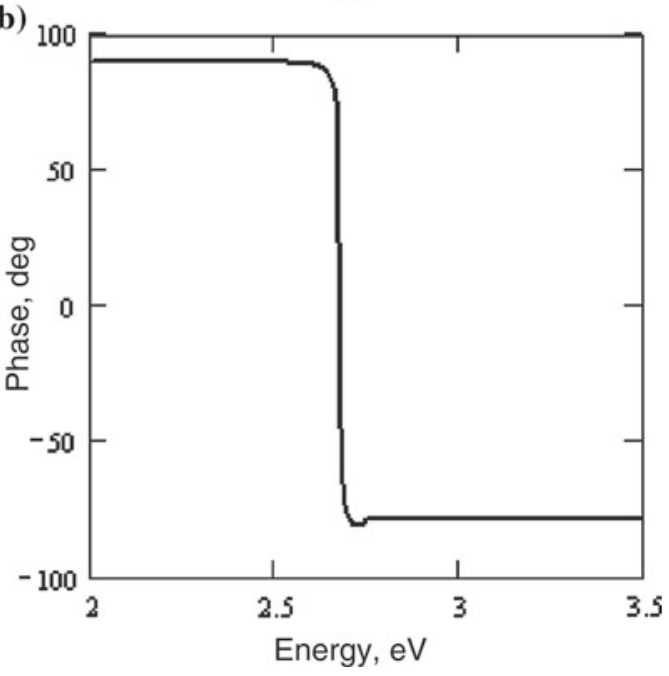

the models, the peak is due to subtracting the components coming from the piston and drum effects in the rear configuration mode. In the phase spectra, this phenomenon is manifested as a change in the zero crossing energy value when the compensation for bending and expansion of the sample due to the heating occurs.

\subsection{Corrections of the Models in the Case of the Presence of Defects}

The above assumptions are sufficient for ideal crystals and surface, but in the case of imperfect materials its modifications must be taken into account. One must consider periodical temperature fields generated in the sample caused by the absorption associated with the defects located in the volume and/or at the surfaces of the samples. 
Fig. 2 Simulation of (a) amplitude and (b) phase spectra for sample of thickness $1 \mathrm{~mm}$, thermal diffusivity of $0.05 \mathrm{~cm}^{2} \cdot \mathrm{s}^{-1}, E_{\mathrm{g}}=2.74 \mathrm{eV}$, thermal conductivity $k=0.19 \mathrm{~W} \cdot \mathrm{cm}^{-1} \cdot \mathrm{K}^{-1}$ with a volume defect of energy $E=2.55 \mathrm{eV}$, $\beta_{\mathrm{d}}=0.05 \mathrm{eV}$, and $A_{\mathrm{d}}=300 \mathrm{~cm}^{-1}$

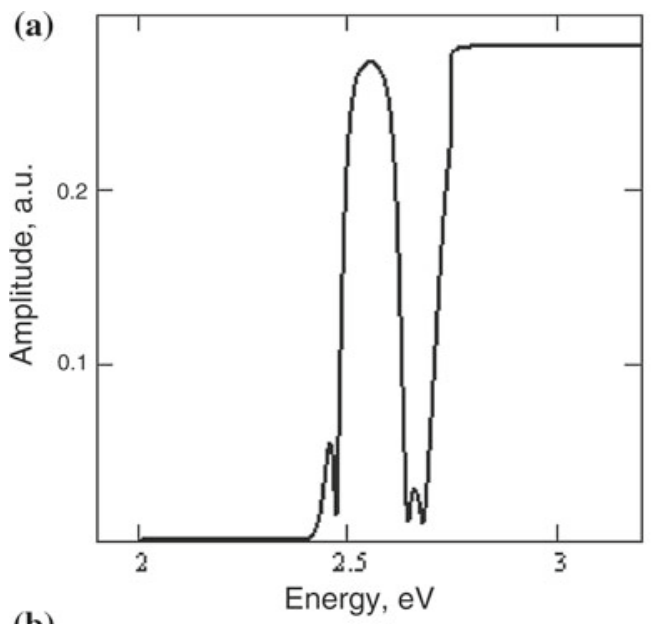

(b)

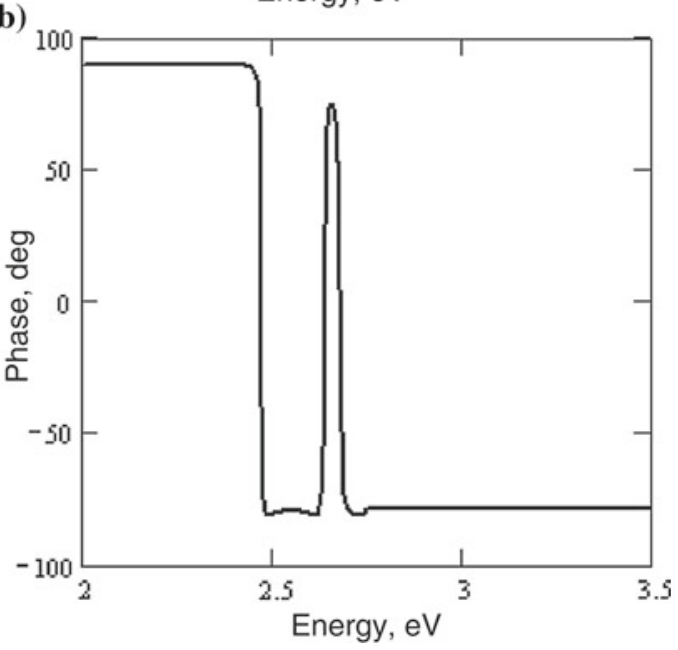

The absorption coefficient due to the presence of the defects has a Gaussian character:

$$
\beta=A_{\mathrm{d}} \exp \left(\frac{E-E_{\mathrm{d}}}{\beta_{1}}\right)^{2}
$$

where $E_{\mathrm{d}}$ is the value of the energy of the defect, $\beta_{1}$ is the parameter describing the width of the Gaussian shape maximum, and $A_{\mathrm{d}}$ is the amplitude of the maximum.

The presence of the defect in the volume introduces an additional maximum in the amplitude and changes the phase of the spectra.

Figure 2 presents the (a) amplitude and (b) phase spectra for a sample of a thickness of $1 \mathrm{~mm}$, a thermal diffusivity of $0.05 \mathrm{~cm}^{2} \cdot \mathrm{s}^{-1}, E_{\mathrm{g}}=2.74 \mathrm{eV}$, a thermal conductivity of $k=0.19 \mathrm{~W} \cdot \mathrm{cm}^{-1} \cdot \mathrm{K}^{-1}$ with a volume defect of energy $E=2.55 \mathrm{eV}, \beta_{\mathrm{d}}=$ $0.05 \mathrm{eV}$, and $A_{\mathrm{d}}=300 \mathrm{~cm}^{-1}$. 
The defect can also be located at the surfaces of the sample, and its nature can be associated with the quality of the surface after the preparation process (grounding, polishing, etching). Malinski [15] proposed an approximate model of thermal distribution in this case. He assumed the presence of a very thin layer of thickness $d$ and thermal parameters $\left(\sigma_{\mathrm{c}}, k_{\mathrm{c}}\right)$ different from the volume of the sample. The presence of the surface defect level of the energy $E_{\mathrm{d}}$ (described by Eq. 11) causes absorption only in a thin layer; the volume of the sample is transparent for this radiation. The temperature in this layer has the form,

$$
T_{\mathrm{d}}(x)=\frac{I_{0}\left(1-\exp (-\beta d)\left[\exp \left(-\sigma_{\mathrm{c}} x\right)+\exp \left(-2 \sigma l+\sigma_{\mathrm{c}} x\right)\right]\right.}{k_{\mathrm{c}} \sigma_{\mathrm{c}}\left(1-\exp \left(-2 \sigma_{\mathrm{c}} l\right)\right)}
$$

and the temperature distribution in the sample is the sum of temperatures in the surface and volume of the sample

$$
T^{\prime}(x)=T(x)+T_{\mathrm{d}}(x)
$$

where $T(x)$ is described by Eq. 7 .

An analogous expression to Eq. 12 can also be obtained for Blonskij's model taking the above given assumptions [16]. Putting the corrected expression for the temperature distribution (Eq. 13) into Eq. 8, one can obtain the theoretical piezoelectric spectra.

Figure 3 shows the amplitude and phase spectra (the same results are obtained for interference and Blonskij's model) for the same condition as in the case of the volume defect; the thermal conductivity of the sample $k_{\mathrm{s}}=0.19 \mathrm{~W} \cdot \mathrm{cm}^{-1} \cdot \mathrm{K}^{-1}$, and for the defected layer, $k_{\mathrm{c}}=0.10 \mathrm{~W} \cdot \mathrm{cm}^{-1} \cdot \mathrm{K}^{-1}$

The different characters of the changes in amplitude and phase are clearly visible in comparison to the simulation of the volume defect. The comparison of the characters of the spectra gives the information not only about the position of the defect but also about its nature.

\subsection{Fernelius Model}

Modifications of interference and Blonskij models due to the influence of surface defects are approximate ones. They can be verified by a comparison to the model based on the conditions of Fernelius [11], whose model is the development of the Rosencwaig and Gersho [10] theory for the case of a two-layer system (Fig. 4).

The author analyzes the temperature originating from such a system but in the case of microphone detection, i.e., the temperature at the surface at $x=0$. To use the model, one must get the solution of the temperature distribution along the length of the sample $T_{\mathrm{S}}(x)$ and coating $T_{\mathrm{c}}(x)$ :

$$
\begin{aligned}
T_{\mathrm{s}}(x)= & U \exp \left(\sigma_{\mathrm{s}} x\right)+V \exp \left(-\sigma_{\mathrm{s}} x\right)-E \exp (\beta x), \\
& -l \leq x \leq 0 \\
T_{\mathrm{c}}(x)= & X \exp \left[\sigma_{\mathrm{c}}(h-x)\right]+Y \exp \left[-\sigma_{\mathrm{c}}(h-x)\right]-Z \exp \left[\beta_{\mathrm{c}}(x-h)\right] \\
& 0 \leq x \leq h
\end{aligned}
$$


Fig. 3 Simulation of amplitude and phase spectra for a sample of thermal conductivity $k_{\mathrm{S}}=0.19 \mathrm{~W} \cdot \mathrm{cm}^{-1} \cdot \mathrm{K}^{-1}$ with a surface defect of energy $E=2.55 \mathrm{eV}$ and $k_{\mathrm{d}}=0.10 \mathrm{~W} \cdot \mathrm{cm}^{-1} \cdot \mathrm{K}^{-1}$

Fig. 4 Cross-sectional view of Fernelius' sample-coating gas setup
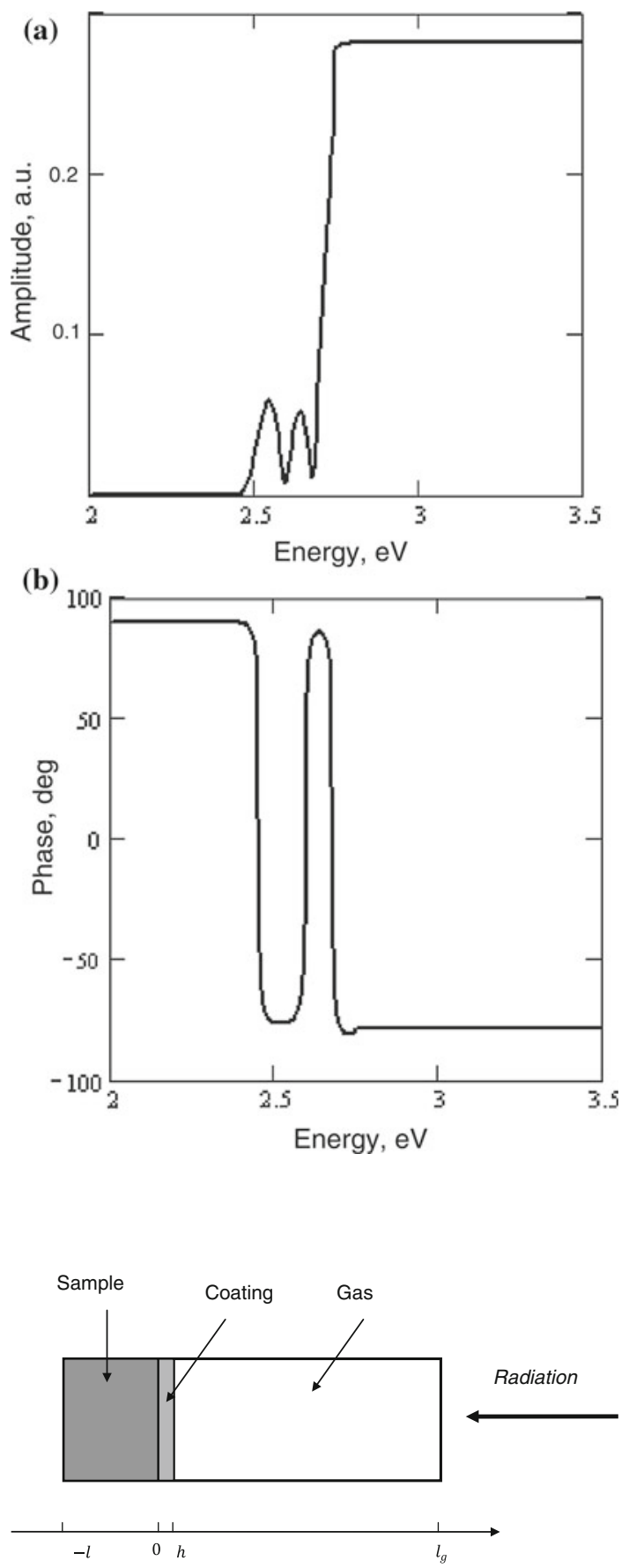
To get the values of $U, V, E$, and $X, Y, Z$ parameters, one must solve the system of equations based on the boundary conditions of the temperature continuity:

$$
\begin{aligned}
T_{\mathrm{g}}(h, t) & =T_{\mathrm{c}}(h, t), \\
T_{\mathrm{c}}(0, t) & =T_{\mathrm{s}}(0, t), \\
T_{\mathrm{b}}(-l, t) & =T(-l, t)
\end{aligned}
$$

and the heat flux continuity:

$$
\begin{aligned}
k_{\mathrm{g}} \frac{\partial T_{\mathrm{g}}(h, t)}{\partial x} & =k_{\mathrm{c}} \frac{\partial T_{\mathrm{c}}(h, t)}{\partial x} \\
k_{\mathrm{c}} \frac{\partial T_{\mathrm{c}}(0, t)}{\partial x} & =k_{\mathrm{s}} \frac{\partial T_{\mathrm{s}}(0, t)}{\partial x} \\
k_{\mathrm{b}} \frac{\partial T_{\mathrm{b}}(-l, t)}{\partial x} & =k_{\mathrm{s}} \frac{\partial T_{\mathrm{s}}(-l, t)}{\partial x}
\end{aligned}
$$

Hence, the system of equations has the form

$$
\begin{aligned}
& \Theta=X+Y-Z \\
& X \exp \left(\sigma_{\mathrm{c}} h\right)+Y \exp \left(-\sigma_{\mathrm{c}} h\right)-Z \exp \left(-\beta_{\mathrm{c}} h\right)=U+V-E \\
& U \exp \left(-\sigma_{\mathrm{s}} l\right)+V \exp \left(\sigma_{\mathrm{s}} l\right)-E \exp (-\beta l)=W \\
& -g \Theta=-c X+c Y-r_{\mathrm{c}} Z \\
& -c X \exp \left(-\sigma_{\mathrm{c}} h\right)+c Y \exp \left(-\sigma_{\mathrm{c}} h\right)-r_{\mathrm{c}} Z \exp \left(-\beta_{\mathrm{c}} h\right)=U-V-r_{\mathrm{s}} E \\
& U \exp \left(-\sigma_{\mathrm{s}} l\right)+V \exp \left(\sigma_{\mathrm{s}} l\right)-r_{\mathrm{s}} E \exp (-\beta l)=b W
\end{aligned}
$$

where $c=\frac{k_{\mathrm{c}} \sigma_{\mathrm{c}}}{k_{\mathrm{s}} \sigma_{\mathrm{s}}}, b=\frac{k_{\mathrm{b}} \sigma_{\mathrm{b}}}{k_{\mathrm{s}} \sigma_{\mathrm{s}}}, g=\frac{k_{\mathrm{g}} \sigma_{\mathrm{g}}}{k_{\mathrm{s}} \sigma_{\mathrm{s}}}, r_{\mathrm{s}}=\frac{\beta}{\sigma_{s}}, r_{\mathrm{c}}=\frac{\beta_{c} k_{\mathrm{c}}}{k_{\mathrm{s}} \sigma_{\mathrm{s}}}$, and $\sigma_{i}=\frac{\mathrm{i} \omega}{\alpha_{i}} ; k_{i}$ and $\alpha_{i}$ are the thermal conductivity and thermal diffusivity of the sample (s), coating (c), backing (b), and gas (g).

Solving the system of Eq. 17 and applying the parameters for Eq. 14, one obtains the temperature along the sample:

$$
\text { where } T(x)=\left\{\begin{array}{l}
T_{\mathrm{s}}(x) \text { for }-l \leq x \leq 0 \\
T_{\mathrm{c}}(x) \text { for } 0 \leq x \leq h
\end{array}\right.
$$

Averaging the temperature at the $Y Z$ plane and using the solution for thermoelastic theory, one gets the expression for the piezoelectric signal,

$$
V=-\left(\frac{1}{l} \int_{-l}^{h} T(x) \mathrm{d} x-\frac{10}{2 r^{2}} \int_{-l}^{h}\left(x+\frac{l-h}{2}\right) T(x) \mathrm{d} x\right)
$$

Using $h=0, k_{\mathrm{s}}=k_{\mathrm{c}}$, and $\alpha_{\mathrm{s}}=\alpha_{\mathrm{c}}$, one obtains the solution for a one-layer system. For these conditions, the amplitude and phase spectra are the same as the ones obtained 
Fig. 5 Comparison of interference/Blosnkij (solid line) and Fernelius (dashed line) models for the case of the presence of a surface defect of energy $2.55 \mathrm{eV}$
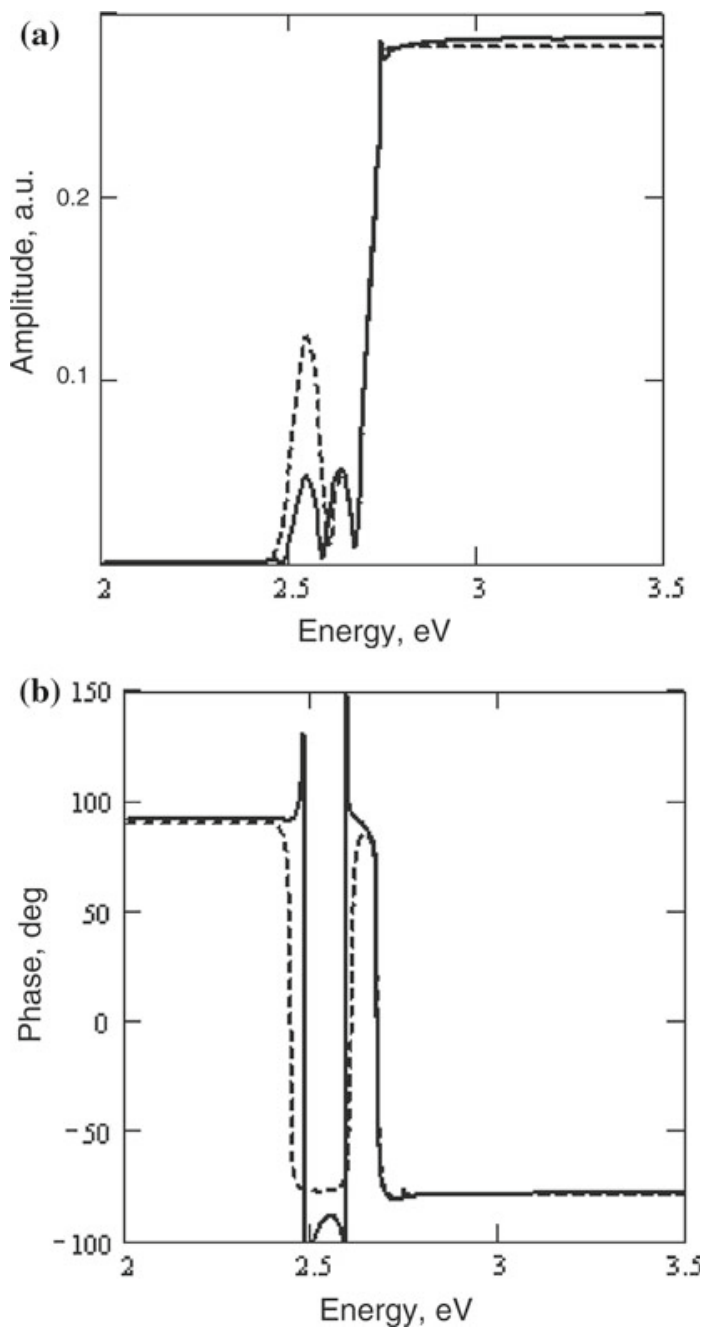

for the interference and Blonskij's models. The same results are also obtained for the presence of the defect in the volume of the sample.

The comparison of the interference/Blosnkij (solid line) and Fernelius (dashed line) models in the case of the presence of a surface defect of energy $2.55 \mathrm{eV}$ are presented in Fig. 5. The thickness of the layer (coating) is $0.01 \mathrm{~mm}$. The other parameters are the same as in Fig. 3. The character of the (a) amplitude and (b) phase are very similar. The intensity of the amplitude maximum at $2.55 \mathrm{eV}$ is higher for the Fernelius model. The changes in phase are observed for the same values of energy. Although the approximated models give the same qualitative results, they must be calibrated to get the quantitative ones. 


\section{Conclusions}

We propose the application of thermal distributions in the interpretation of amplitude and phase spectra of a piezoelectric signal. For the first time, the full solution of a two-layer model was used and it was compared to the interference model and the model was proposed by Blonskij.

All of them can be applied to simulate the amplitude and phase piezoelectric spectra. They give the same results for ideal semiconducting crystals and in the case of defects located in the volume of the sample. Additional assumptions were introduced to simulate the surface defects. The volume and surface defects manifest their presence in different ways, giving unlike changes in amplitude and phase spectra. Their comparison can be applied to determine their nature.

The models based on interference and Blonskij's theories must be calibrated to obtain quantitative results. Comparison and fitting to the experiment results should enable one to determine optical (energy gap) and thermal parameters (thermal diffusivity, thermal conductivity) of the investigated materials. The thickness and thermal parameters of the destroyed layer can also be estimated after comparison to the other methods.

Open Access This article is distributed under the terms of the Creative Commons Attribution License which permits any use, distribution, and reproduction in any medium, provided the original author(s) and the source are credited.

\section{References}

1. P. Wang, T. Nakagawa, A. Fukuyama, K. Maeda, Y. Iwasa, M. Ozeki, Y. Akashi, T. Ikari, Sci. Eng. C 26, 826 (2006)

2. A. Fukuyama, S. Sakamoto, S. Sonoda, P. Wang, K. Sakai, T. Ikari, Thin Solid Films 112, 511 (2006)

3. K. Sakai, T. Kakeno, T. Ikari, S. Shirakata, T. Sakemi, K. Awai, T. Yamamoto, J. Appl. Phys. 99, 043508 (2006)

4. K. Yoshino, H. Komaki, T. Kakeno, Y. Akaki, T. Ikari, J. Phys. Chem. Solids 64, 1839 (2003)

5. T. Hoshimiya, NDT \& E Int. 32, 133 (1999)

6. W. Jackson, N.M. Amer, J. Appl. Phys. 51, 3343 (1980)

7. I.V. Blonskij, V.A. Thoryk, M.L. Shendeleva, J. Appl. Phys. 79, 3512 (1996)

8. C. Bennett Jr., R.R. Patty, Appl. Opt. 21, 49 (1982)

9. M. Maliński, Arch. Acoust. 27, 217 (2002)

10. A. Rosencwaig, A. Gersho, J. Appl. Phys. 47, 64 (1976)

11. N. Fernelius, J. App. Phys. 51, 650 (1980)

12. S.P. Timoshenko, J.N. Goodier, Theory of Elasticity (McGraw-Hill, New York, 1970)

13. A.M. Katz, Theory of Elasticity (GITTL, Moscow, 1956)

14. J.I. Pankove, Optical Processes in Semiconductors (Dover, New York, 1976)

15. M. Maliński, K. Zakrzewski, J. Strzałkowski, Int. J. Thermophys. 28, 299 (2007)

16. J. Zakrzewski, M. Maliński, K. Strzałkowski, D. Madaj, F. Firszt, S. Łęgowski, H. Męczyńska, Int. J. Thermophys. 33, 733 (2012) 